\title{
Incidence of thromboembolic complications in patients with mechanical heart valves with a subtherapeutic international normalized ratio
}

Francesco Dentali, MD, ${ }^{\mathrm{a}}$ Nicoletta Riva, MD, ${ }^{\mathrm{a}}$ Alessandra Malato, MD, ${ }^{\mathrm{b}}$ Giorgia Saccullo, MD, ${ }^{\mathrm{b}}$ Sergio Siragusa, MD, ${ }^{\mathrm{b}}$ and Walter Ageno, $\mathrm{MD}^{\mathrm{a}}$

Objective: Subtherapeutic international normalized ratios are frequently encountered in clinical practice, and patients with mechanical heart valves with inadequate anticoagulation may be exposed to an increased risk of thromboembolic events. There are no data on thromboembolic event risk for these patients.

Methods: We assessed the current practice patterns in the management of patients with mechanical heart valves with subtherapeutic international normalized ratios and assessed the risk of thromboembolic complications in this setting. The charts of patients with mechanical heart valves followed up in two anticoagulation clinics were reviewed. Patients with a history of stable, therapeutic anticoagulation but with a subtherapeutic international normalized ratio were included. Patients who underwent invasive procedures requiring temporary suspension of antithrombotic therapy were excluded. Data on use and dose of low-molecular weight heparin bridging therapy were collected.

Results: The incidence of objectively confirmed thromboembolic events within 90 days after obtaining the index international normalized ratio was assessed. Two hundred ninety-four patients with mechanical heart valves were included (mean age 63.3 years, $47.3 \%$ male). Low-molecular weight heparin was prescribed in 14 cases $(4.8 \%)$. At 90 days, 1 patient had a thromboembolic complication $(0.3 \%, 95 \%$ confidence interval $0 \%-1.9 \%)$.

Conclusion: Patients with previously stable, therapeutic anticoagulation with a subtherapeutic international normalized ratio have a low risk of thromboembolic events. Withholding low-molecular weight heparin bridging therapy is a reasonable therapeutic option in these cases.

Patients with mechanical prosthetic heart valves require long-term antithrombotic prophylaxis with vitamin $\mathrm{K}$ antagonists (VKAs) to reduce the risk of thromboembolic complications. In the absence of anticoagulant therapy, the estimated risk of thromboembolic events for patients with a prosthetic valve in the aortic position is about $12 \%$ per year rising to $22 \%$ per year for patients with a prosthetic valve in the mitral position. ${ }^{1,2}$ The American College of Chest Physicians guidelines recommend long-term anticoagulant therapy with a target international normalized ratio (INR) value of 2.5 (range 2.0-3.0) or 3.0 (range 2.5-3.5) for patients with mechanical prosthetic heart valves, ${ }^{3}$ depending on the position, the type of valve, and the presence of additional risk factors. The association of VKAs with aspirin is recommended for patients at very high risk (patients with caged-ball or caged-disk valves or additional risk factors, such as atrial fibrillation or myocardial infarction) and in case of systemic embolism despite therapeutic INR values. ${ }^{3}$

\footnotetext{
From the Department of Clinical Medicine, University of Insubria, Varese, Italy, and the Thrombosis/Haemostasis and Haematology Unit, University Hospital of Palermo, Palermo, Italy. ${ }^{\mathrm{b}}$

Received for publication Jan 21, 2008; revisions received May 25, 2008; accepted for publication July 15,2008

Address for reprints: Francesco Dentali, MD, U.O. Medicina I, Ospedale di Circolo, Viale Borri 57, 21100 Varese, Italy (E-mail: fdentali@libero.it).

J Thorac Cardiovasc Surg 2009; 137:91-3

$0022-5223 / \$ 36.00$

Copyright $(c) 2009$ by The American Association for Thoracic Surgery

doi:10.1016/j.jtcvs.2008.06.030
}

Subtherapeutic INR values are frequently encountered in clinical practice, and patients with mechanical heart valves with inadequate anticoagulation may be exposed to an increased risk of thromboembolic events. The current literature, however, inadequately describes the clinical consequences associated with exposure to subtherapeutic INR values.

Bridging therapy with low-molecular weight heparin (LMWH) has been shown to be effective and relatively safe for patients with mechanical prosthetic heart valves who require interruption of VKAs for an invasive procedure. ${ }^{4-6}$ Whether administration of LMWH is also necessary to prevent thromboembolic complications in patients with subtherapeutic INR values is currently unknown. Before a clinical trial assessing the efficacy and safety of LMWH in this setting is eventually planned, however, better information on the actual risk of thromboembolic events for these patients is required. The purposes of this retrospective cohort study were to assess current practice patterns in the management of patients with mechanical prosthetic heart valves with subtherapeutic INR values and to asses the risk of thromboembolic complications in this setting.

\section{MATERIALS AND METHODS}

The charts of all patients with mechanical heart valves who were currently attending or who had attended two anticoagulation clinics in Varese and Palermo, Italy, were reviewed. At both centers, patients are regularly followed up for the monitoring of VKA therapy, and information on clinical events is documented and registered in a computerized database. 


\section{Abbreviations and Acronyms \\ INR = international normalized ratio \\ LMWH $=$ low-molecular weight heparin \\ VKA $=$ vitamin $\mathrm{K}$ antagonist}

Patients were included if they had at least 1 INR value 0.5 to 1 INR units below the lower limit of the patient-specific target INR range, the 2 INR values preceding the index INR value were within or greater than the patient-specific INR target range, and the interval between the 2 INR values preceding the index INR value was at least 2 weeks. Because risk of thromboembolic events is highest in the first 3 months after surgery, only patients with anticoagulation for more than 3 months were included. Patients who underwent invasive procedures requiring the temporary suspension of the antithrombotic therapy were excluded.

The INR value and baseline characteristics, including age, sex, position (mitral or aortic) and type (caged-ball, caged-disk, tilting-disk, or bileaflet) of mechanical valve, target therapeutic INR range, concomitant therapy with aspirin, presence of atrial fibrillation, presence of left atrial dilatation, presence of heart failure with low ejection fraction, history of stroke or transient ischemic attack, history of myocardial infarction, were registered anonymously in a database with a patient number as the only identifier. The use and dose of LMWH bridging therapy, were also collected. At each ambulatory follow-up visit, patients were routinely asked about thromboembolic complications. Patients absent at a planned ambulatory visit were contacted by phone; if possible, a new ambulatory visit was planned. Documentation was requested when a possible thromboembolic complication was suspected. For each patient included in the study, the occurrence of thromboembolic events within 90 days after the index INR value was assessed. Only objectively confirmed events were considered.

The primary outcome was the proportion of patients with thromboembolic complications (stroke, transient ischemic attack, peripheral embolism, symptomatic valve thrombosis) within 90 days after the index INR value was obtained. The incidences of thromboembolic complications among patients with and without prophylaxis were also separately analyzed. Source documents of clinical events were requested and evaluated by 2 investigators (F.D., A.M.).

Finally, the rate of major bleeding events was also assessed. Patient characteristics were reported as mean $\pm \mathrm{SD}$ and proportion. The $95 \%$ confidence intervals were calculated for the end points of the study. The study was approved by the local ethics committees.

\section{RESULTS}

Baseline characteristics are summarized in Table 1. Agreement between the 2 investigators in reviewing patient charts was perfect. Three hundred twelve consecutive patients with mechanical heart valves were identified. Eighteen did not satisfy inclusion criteria ( 3 had started oral anticoagulation less than 3 months previously, 15 did not have any INR value $0.5-1$ INR units below the lower limit of the patient-specific target INR range with the 2 preceding INR values within or greater than the patient-specific INR target range), leaving 294 patients eligible for the analysis. Mean age of included patients was $63.3 \pm 11.9$ years (range 19-79 years), and 139 were male $(47.3 \%)$. One hundred twenty-nine patients had aortic mechanical heart valves $(43.9 \%), 114$ had mitral mechanical heart valves $(39.1 \%)$, and 50 had both mitral and aortic mechanical heart valves
TABLE 1. Baseline characteristics

\begin{tabular}{lc}
\hline Patients (No.) & 294 \\
Age (y, mean \pm SD) & $63.3(11.9 \%)$ \\
Male sex (No.) & $139(47.3 \%)$ \\
Valve position (No.) & \\
$\quad$ Aortic & $129(43.9 \%)$ \\
$\quad$ Mitral & $115(39.1 \%)$ \\
$\quad$ Mitroaortic & $50(17.0 \%)$ \\
Type of valve (No.) & \\
$\quad$ Bileaflet & $283(96.3 \%)$ \\
$\quad$ Caged-disk or tilting-disk & $11(3.7 \%)$ \\
Additional risk factors (\%) & \\
$\quad$ Atrial fibrillation & $33.3 \%$ \\
$\quad$ Atrial enlargement & $15.5 \%$ \\
Coronary heart disease & $17.4 \%$ \\
$\quad$ Cerebrovascular disease & $13.2 \%$ \\
$\quad$ Heart failure & $14.0 \%$ \\
INR value at presentation (mean \pm SD) & $1.74 \pm 0.17$ \\
Time to next INR measurement (d, mean \pm SD) & $10.3 \pm 4.7$ \\
Patients with 1 or more subtherapeutic INR values (No.) & $138(46.9 \%)$ \\
Duration of subtherapeutic anticoagulation & $16.3 \pm 9.0$ \\
$\quad$ (d, mean \pm SD) & \\
\hline INR, International normalized ratio. &
\end{tabular}

$(17.0 \%)$. Almost all patients had bileaflet mechanical heart valves. One third of patients had concomitant atrial fibrillation, $15.5 \%$ had atrial enlargement without fibrillation, $17.4 \%$ had a history of myocardial infarction, $13.2 \%$ had a previous stroke or transient ischemic attack, and $14.0 \%$ had low ejection fraction. More than $60 \%$ of patients had at least 1 additional risk factor.

Concomitant antiplatelet therapy with aspirin was used in 20 cases $(6.8 \%)$. Mean INR value at the time of the presentation was $1.74 \pm 0.17$, and $84 \%$ of patients had a new INR value determined within 2 weeks. Subsequent INR value was considered subtherapeutic in 138 patients (46.9\%), and mean duration of subtherapeutic anticoagulation was 16.3 days \pm 9.0 . LMWH was prescribed for 14 patients $(4.8 \%)$. A prophylactic dose was used in 6 cases and a therapeutic dose in 8 cases.

All patients had a follow-up of at least 90 days. During the 90 days of follow-up, only 1 patient had a thromboembolic complication $(0.3 \% ; 95 \%$ confidence interval $0 \%-1.9 \%$; 1.45 events for 100 patients per year). In a patient with bileaflet mitral and aortic mechanical heart valves and concomitant atrial fibrillation, a stroke occurred 8 days after the index INR value was obtained.

When we consider only patients who did not receive bridging therapy with $\mathrm{LMWH}$, the incidence of thromboembolic complication was $0.4 \%$ (95\% confidence interval $0 \%-2.0 \%$ ). There were no major bleeding events during the period of observation.

\section{DISCUSSION}

The results of our study suggest that patients with mechanical heart valves with previously stable, therapeutic 
anticoagulation who have a subtherapeutic INR value $(0.5-$ 1.0 INR units below the lower limit of the patient-specific target INR range) are at low risk for thromboembolic events in the ensuing 90 days. The validity of our results is strengthened by the characteristics of the included population, because more than $50 \%$ of our patients had a mitral or mitroaortic mechanical heart valve, and more than $60 \%$ had at least one additional risk factor and therefore could be considered at high risk for thromboembolic complications. Furthermore, the annual risk of thromboembolic events in our cohort of patients not receiving LMWH (1.45 events per 100 patients per year) did not seem to be higher than the annual risk or thromboembolic events reported in other studies, including those of patients receiving long-term anticoagulation for mechanical heart valves. ${ }^{3,7}$

These findings may have clinical importance, since bridging therapy with LMWH while awaiting the return of the INR value to the desired range appears to be unnecessary for these patients. This conclusion is supported on the one hand by the low observed incidence of thromboembolic events and on the other hand by the potentially increased risk of bleeding complications and by the cost associated with the additional treatment with LMWH.

These results are in agreement with the results of a casecontrol study in which the incidence of complications related to anticoagulation did not differ between a large group of patients with long-term anticoagulation whose INR values were at least 0.5 units below the bottom end of the therapeutic INR range and a group of patients with similar characteristics whose INR values were within the patient-specific therapeutic range. ${ }^{8}$ Only a minority of patients included in that study, however, had prosthetic heart valves, and thus no conclusions for such patients could be drawn in that setting.

Validity of observational retrospective design is a potential limitation of our study. To avoid misleading results, we paid meticulous attention to our ascertainment of all thromboembolic events by reviewing ambulatory charts of all included patients and by contacting patients without complete ambulatory charts for follow-up. Absence of a control group is another potential limitation. The number of thromboembolic events was extremely low in our series of patients, however, and such events remained uncommon even when the upper limits of the confidence intervals of our results were considered. Finally, the number of patients receiving LMWH was small, and conclusions about this subgroup are therefore of limited value.

In conclusion, patients with mechanical heart valves and previously stable therapeutic anticoagulation who have a subtherapeutic INR value have a low risk of subsequent thromboembolic events in the ensuing 90 days. Withholding bridging therapy with LMWH while awaiting return of the INR value to the desired range appears to a reasonable therapeutic option in these cases. Larger prospective studies are warranted to confirm our preliminary results.

\section{References}

1. Baudet EM, Puel V, McBride JT, Grimaud JP, Roques F, Clerc F, et al. Long-term results of valve replacement with the St. Jude Medical prosthesis. J Thorac Cardiovasc Surg. 1995;109:858-70.

2. Cannegieter SC, Rosendaal FR, Wintzen AR, Van Der Meer FJ, Vandenbroucke JP, Briët E. Optimal oral anticoagulant therapy in patients with mechanical heart valves. $N$ Engl J Med. 1995;333:11-7.

3. Salem DN, Stein PD, Al-Ahmad A, Bussey HI, Horstkotte D, Miller N, et al. An tithrombotic therapy in valvular heart disease-native and prosthetic: the Seventh ACCP Conference on Antithrombotic and Thrombolytic Therapy. Chest. 2004; 126(3 Suppl):457S-82S.

4. Ansell J, Hirsh J, Poller L, Bussey H, Jacobson A, Hylek E. The pharmacology and management of the vitamin K antagonists: the Seventh ACCP Conference on Antithrombotic and Thrombolytic Therapy. Chest. 2004;126(3 Suppl):204S-33S.

5. Ferreira I, Dos L, Tornos P, Nicolau I, Permanyer-Miralda G, Soler-Soler J. Experience with enoxaparin in patients with mechanical heart valves who must withhold acenocumarol. Heart. 2003;89:527-30.

6. Hammerstingl C, Tripp C, Schmidt H, von der Recke G, Omran H. Periprocedural bridging therapy with low-molecular-weight heparin in chronically anticoagulated patients with prosthetic mechanical heart valves: experience in 116 patients from the prospective BRAVE registry. J Heart Valve Dis. 2007;16:285-92.

7. Pengo V, Barbero F, Banzato A, Garelli E, Noventa F, Biasiolo A, et al. A comparison of a moderate with moderate-high intensity oral anticoagulant treatment in patients with mechanical heart valve prostheses. Thromb Haemost. 1997;77:839-44.

8. Clark N, Witt D, Delate T, Trapp M, Garcia D, Ageno W, et al. Thromboembolic consequences of subtherapeutic anticoagulation in patients stabilized on warfarin therapy: the low INR Study. Pharmacotherapy. 2008;28(8):960-7. 